Articles

\title{
REM Sleep Behavior and Motor Findings in Parkinson's Disease: A Cross- sectional Analysis
}

\author{
Abhimanyu Mahajan ${ }^{1}$, Liana S. Rosenthal ${ }^{1}$, Charlene Gamaldo ${ }^{1}$, Rachel E. Salas ${ }^{1}$, Gregory M. Pontone ${ }^{2}$, Arita McCoy $^{1}$, Chizoba Umeh ${ }^{1}$ \& \\ Zoltan Mari ${ }^{*}$ \\ ${ }^{1}$ Department of Neurology Johns Hopkins University, Baltimore, MD, USA, ${ }^{2}$ Department of Psychiatry and Behavioral Sciences Johns Hopkins University, \\ Baltimore, MD, USA
}

\begin{abstract}
Background: Parkinson's disease (PD) represents a major public health challenge that will only grow in our aging population. Understanding the connection between PD and associated prodromal conditions, such as rapid eye movement sleep behavioral disorder (RBD), is critical to identifying prevention strategies. However, the relationship between RBD and severity of motor findings in early PD is unknown. This study aims to examine this relationship.

Methods: The study population consisted of 418 PD patients who completed the Movement Disorders Society-United Parkinson's Disease Rating Scale (MDSUPDRS) and rapid eye movement sleep (REM) disorder questionnaires at the baseline visit of the Michael J. Fox's Parkinson's Progression Markers Initiative (PPMI). Cross-sectional analysis was carried out to assess the association between REM Sleep Behavior Screening Questionnaire score and MDS UPDRS-3 (motor) score categories. Correlation with a higher score category was described as "worse motor findings". A score of 5 on the REM disorder questionnaire was defined as predictive of RBD.
\end{abstract}

Results: Out of the 418 PD patients, $113(27.0 \%)$ had RBD. With univariate logistic regression analysis, individuals with scores predictive of RBD were 1.66 times more likely to have worse motor findings $(\mathrm{p}=0.028)$. Even with age, gender, and Geriatric Depression Scale scores taken into account, individuals with scores predictive of RBD were 1.69 times more likely to have worse motor findings $(\mathrm{p}=0.025)$.

Discussion: PD patients with RBD symptoms had worse motor findings than those unlikely to have RBD. This association provides further evidence for the relationship between RBD and PD.

Keywords: Parkinson's disease, rapid eye movement sleep, motor findings, Movement Disorders Society-United Parkinson's Disease Rating Scale Citation: Mahajan A, Rosenthal LS, Gamaldo C, et al. REM sleep behavior and motor findings in Parkinson's disease: A cross-sectional analysis. Tremor Other Hyperkinet Mov. 2014; 4. doi: 10.7916/D84B2ZDF

* To whom correspondence should be addressed. E-mail: zmari1@jhmi.edu

Editor: Elan D. Louis, Columbia University, USA

Received: April 16, 2014 Accepted: May 20, 2014 Published: June 23, 2014

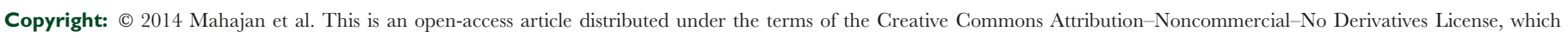

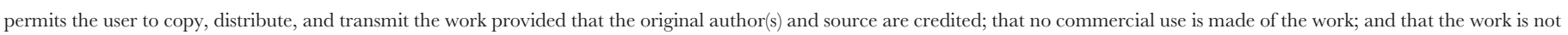
altered or transformed.

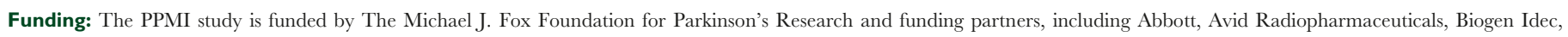
Covance, Elan Corporation, plc, GE Healthcare, Genentech, GlaxoSmithKline, Eli Lilly and Company, Merck, Pfizer Inc., Roche CNS group, and UCB.

Financial Disclosures: None.

Conflict of Interest: The authors report no conflict of interest.

Disclaimer: The views expressed in the submitted article by the authors are their own and not an official position of the institution or funder.

\section{Introduction}

As medical advances extend human life, the prevalence of age-related diseases, including Parkinson's disease (PD) will increase worldwide. ${ }^{1}$ Motor disability has been considered the hallmark of PD and a standard measure of disease severity, particularly when no PD medications confound the rating. Mobility and motor findings are an important determinant of PD patients' functionality and therefore highly relevant to their health status. ${ }^{2}$ Symptomatic therapies are readily available for PD with overall excellent results, yet they fail to prevent or delay disease progression and are limited in improving patients' quality of life in late stages of the disease. With only preventative or disease-modifying therapies offering the prospect of a profound and lasting impact on PD patients' lives, interventions aimed at the disease process and applied before the motor findings manifest are extremely appealing. 
Prodromal manifestations predate the appearance of motor symptoms often by years. Specifically, there is now direct evidence that certain prodromal symptoms, such as anosmia, rapid eye movement (REM) sleep behavior disorder (RBD), constipation, and depression can be present in the prodromal phase of PD. ${ }^{3}$ Understanding the connection between PD and prodromal conditions, such as RBD, is critical to research efforts aimed at prevention and disease modification.

$\mathrm{RBD}$ is a parasomnia manifested by vivid, often frightening dreams associated with simple or complex motor behavior during REM sleep. ${ }^{4}$ Fulda et al. ${ }^{5}$ reported that RBD may be one of the earliest signs of and/or a long-term predictor for neurodegenerative disorders, particularly PD. ${ }^{5} \mathrm{RBD}$ and stage 2 of $\mathrm{PD}$ (i.e., the stage of disease when PD symptoms are bilateral, affecting both limbs and both sides of the body), both show pathomorphological similarity by way of demonstration of alpha-synuclein pathology in the brain, suggesting that RBD is a pathway to PD. ${ }^{5}$ In fact, patients with RBD have up to a $65 \%$ risk of developing $\mathrm{PD}$ at 10 years, making RBD a specific clinical marker of premotor PD. ${ }^{6} \mathrm{RBD}$ has been reported to be present in $42.6 \%$ of all PD patients. ${ }^{7}$ Despite these high percentages, only a few systematic, large-scale studies have investigated the characteristics of RBD in PD. The Parkinson's Progression Marker Initiative (PPMI) is one such effort which encompasses 32 internationally recognized clinical sites that are jointly involved in a comprehensive study designed to identify potential PD progression biomarkers. ${ }^{8}$ The PPMI therefore provides an early opportunity to examine relationships between motor findings and factors which could potentially influence them at the onset of PD in a large, untreated, imaging-confirmed cohort of newly diagnosed PD patients.

Although several studies have examined RBD as a potential prodromal condition for $\mathrm{PD},{ }^{6}$ our analysis specifically seeks to assess the association between $\mathrm{RBD}$ and early-onset $\mathrm{PD}$ motor findings severity.

In this analysis, we aim to evaluate the independent association between RBD and motor findings in PD, accounting for age, gender, and common comorbid PD conditions such as depression. The PPMI study presents a unique opportunity to examine such associations in patients that are newly diagnosed, where medications do not confound motor measures. The presence of an independent association between RBD and motor finding severity in early disease may help further establish the link between prodromal states and classical motor manifestations of PD. Such findings could help advance the understanding of PD's prodromal states, which may lead to preventative therapies.

\section{Methods}

The PPMI study includes a cohort of PD patients and healthy participants enrolled for clinical assessments, imaging data, and biospecimen collection using standardized data acquisition protocols. ${ }^{8}$ The uniqueness of PPMI lies in the fact that it is the largest de novo PD cohort in history. It includes PD patients whose symptoms do not require medications for motor symptoms at the time of enrollment and up to 6 months thereafter (per enrolling investigator's judgment). Furthermore, each of the PPMI cohort's PD patients underwent nuclear imaging to confirm striatal dopamine deficit. ${ }^{8}$ The majority of the patients had DaTscan, which uses ioflupane iodine-123 as a tracer with single-photon emission computed tomography for measuring the dopamine transporter. Australian sites used AV-133, a positron emission tomography tracer [9-[(18) F]fluoropropyl-(+)-dihydrotetrabenazine ([(18)F]AV-133)]. An abnormal scan (either DaTscan or AV133) was an inclusion criterion for the patient group. The Johns Hopkins Medicine Institutional Review Board approved the PPMI study at our site. The data are publically accessible through a standard application process using the PPMI website. Our team was granted such access by the PPMI Data and Publications Committee (DPG). Additionally, our manuscript was submitted to the PPMI DPC and received administrative approval.

A total of 418 participants with complete data on the selected variables were included in this analysis. We present an abbreviated version of the study participant enrollment criteria as it relates to the current data extraction. Please refer to previously published papers for in-depth descriptions of the overarching PPMI longitudinal study methods as well as the inclusion and exclusion criteria. ${ }^{8}$ Data used for this analysis were obtained from the PPMI database (version 08-012013). ${ }^{9}$

The demographics of all study participants were collected at the screening visit. Once enrolled, the participants underwent a series of tests including the Movement Disorders Society-United Parkinson's Disease Rating Scale (MDS-UPDRS) and REM Sleep Behavior Questionnaire. MDS-UPDRS testing was administered by a certified movement disorders expert.

The RBD questionnaire is a validated 10-item patient self-rating questionnaire covering the clinical features of RBD. With a maximum total score of 13 points, a cut-off of 5 is considered suggestive of RBD. ${ }^{10}$ While this measure cannot make the diagnosis of RBD or substitute polysomnography, it was deemed appropriate for the purposes of evaluating a possible association between RBD symptoms and motor manifestations.

For the Geriatric Depression Scale (GDS), a cut-off of 5 was considered suggestive of depression. ${ }^{11}$ We included scores of MDSUPDRS 3 (motor) questionnaire in this study. The MDS-UPDRS scale is a Movement Disorders Society (MDS)-sponsored revision of the widely used UPDRS scale. The original UPDRS placed considerable emphasis on marked and severe disabilities or impairments. The MDS-UPDRS scale may be better tuned for mild impairment. ${ }^{12}$ For the MDS-UPDRS 3 (motor) section of the MDSUPDRS scale, the total score is 120. MDS-UPDRS scores are not linear. In a study evaluating biomarkers of PD, Lin et al. ${ }^{13}$ used a method of grouping UPDRS scores to classify PD severity into categories. For the purpose of clinical significance and applicability, we devised four categories for the severity of motor findings:

- Category MDS-UPDRS III score range

- Very mild: 0-5

- Mild: 6-24 
- Moderate: 25-60

- Severe: $>60$

Finally, an ordered logistic regression model was constructed with age, gender, REM sleep score category, and GDS score category as predictor variables. The outcome variable was MDS-UPDRS III scores, which were categorized as above.

\section{Statistical analysis}

Statistical procedures were performed with STATA, Version SE 12 for Windows (College Station, TX). Significance was set a priori at $\mathrm{p}<0.05$. A two-sided t test was conducted to evaluate the strength of correlation between dependent and independent variables. The outcome variable, MDS-UPDRS III score, was classified into four categories of motor severity (very mild, mild, moderate, and severe). As the outcome variable is ordinal in nature, a likelihood ratio test for proportionality of odds across response categories was conducted and the parallel regression assumption was not found to be violated (prob $>\mathrm{chi}^{2}$ : 0.4119). Ordered logistic regression models, in univariate and multivariate forms were used to investigate the contribution of individual variables to the prediction of motor findings. Based on the ordered regression analysis, correlations were made between the predictor variables and the outcome variable. The correlation of a higher MDS-UPDRS III category with RBD symptomatology was described as "worse motor findings" with RBD symptomatology.

The RBD screening questionnaire score was treated as a binary variable. Participants with a score above 5 were classified as having RBD symptomatology. ${ }^{10}$

\section{Results}

A total of 418 participants with data on RBD questionnaire scores, GDS scores, MDS-UPDRS 3 scores, and demographic variables were analyzed. In this group, $122(29.2 \%)$ were women and $272(65.1 \%)$ were men. The data on gender of 24 participants were missing. The average age of participants was 62.9 years, ranging from 34 years to 86 years of age (Table 1).

For the sake of simplicity, we refer to our arbitrarily defined group with the higher RBD questionnaire score $(>5)$ as "likely RBD", whereas those with low score $(\leq 5)$ on the questionnaire as "unlikely RBD". Of the 418 PD patients included in this analysis, $113(27 \%)$ were classified as PD patients with likely RBD, while 305 (72.9\%) were classified as unlikely RBD.

Two individuals $(0.5 \%)$ were classified with very mild motor findings such as minimal to no bradykinesia, rigidity, postural instability, freezing of gait, resting tremors, etc., 290 (69.4\%) with mild severity of motor findings, and $126(30.1 \%)$ patients with moderate severity of motor findings. There were no participants who were classified with severe motor findings.

In univariate logistic regression analysis, the odds of having worse motor findings were 1.66 times in participants with likely RBD compared with those unlikely to have RBD ( $p=0.028$, 95\% CI: $1.057-$ 2.62). Even when age, gender and GDS scores were taken into account, the association between RBD and MDS-UPDRS 3 score categories was significant $(\mathrm{OR}=1.69,95 \% \mathrm{CI}: 1.07-2.69, \mathrm{p}=0.025)$.

Among patients unlikely to have RBD, $27.2 \%$ have moderate motor findings and $72.1 \%$ have mild motor findings. In patients with likely $\mathrm{RBD}$, this percentage goes up to $38 \%$ for moderate motor findings, with $61.9 \%$ for those with mild motor findings (Figure 1) $(p=0.025)$.

A sub-analysis was conducted using just Question 6 (question on "dream enactment") of the REM sleep behavior screening questionnaire as a binary variable (presence ${ }^{1-4}$ vs. absence [0]) in place of the REM sleep behavior disorder screening questionnaire score in the model. Using Question 6 as a tool for identifying patients with likely $\mathrm{RBD}$, the odds of having worse motor findings in PD patients with likely REM sleep behavior disorder are 0.71 times compared to PD patients unlikely to have REM sleep behavior disorder ( $p=0.115$, 95\% CI: $1.08-$ 2.22). After accounting for age, gender, and GDS scores, the odds of having worse motor findings in PD patients with likely REM sleep behavior disorder are 1.34 times compared to PD patients unlikely to have REM sleep behavior disorder ( $\mathrm{p}=0.171,95 \%$ CI: 0.488-1.135).

\section{Discussion}

Based on the results of this analysis, we report that a RBD questionnaire score of greater than 5, indicative of RBD symptoms, is an independent predictor of worse motor findings in PD patients.

Table 1. Characteristics at Baseline of Participants with Likely RBD and Unlikely RBD

\begin{tabular}{|lccc}
\hline & Patients with Likely RBD (I I3) & Patients with Unlikely RBD (305) & P \\
\hline Gender (\% male) & $82(72.57 \%)$ & $190(62.30 \%)$ & 0.03 \\
$\begin{array}{l}\text { Depression } \\
(\text { GDS }>5)\end{array}$ & $47(33.1 \%)$ & $91(66.9 \%)$ & 0.06 \\
Age & 63.04 years (SD: 9.74$)$ & 62.87 years (SD: 9.76$)$ & 0.85
\end{tabular}

RBD, Rapid Eye Movement Sleep Behavioral Disorder.

Data on bed partners and usage of antidepressants was not a part of this analysis. RBD screening questionnaire scores and MDS UPDRS III scores were categorized for the purpose of analysis. Details have been discussed in the manuscript.

This table compares baseline characteristics between patients with likely RBD and patients with unlikely RBD. Our analysis showed a statistically significant difference in the percentage of males between the two groups. There was no significant difference in the GDS-indicated depression percentage and age between the two groups. 


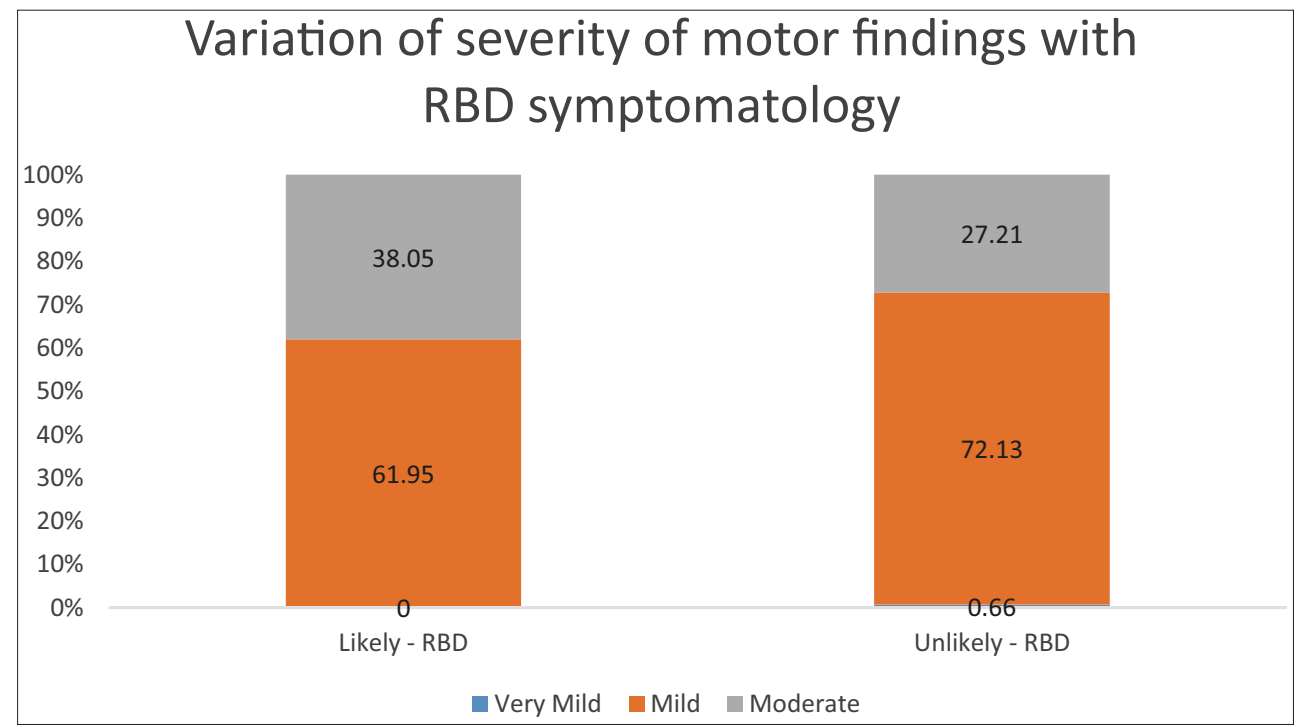

Figure 1. Distribution of Severity of Motor Symptoms in Participants with Likely RBD and unlikely RBD. RBD, rapid eye movement sleep behavioral disorder.

This is the first study to our knowledge evaluating the relationship between RBD symptoms and motor severity within a large de novo, imaging-confirmed PD cohort. We found the odds of having worse motor findings were 1.66 times greater in patients with likely RBD than those unlikely to have RBD $(p=0.028)$. After age, gender, and GDS scores were taken into account, the odds of having worse motor findings were 1.69 times greater in patients with potential RBD than those likely without RBD ( $\mathrm{p}=0.025)$. Among patients unlikely to have RBD, $27.2 \%$ had moderate motor findings, and this percentage went up to $38 \%$ for patients with $\operatorname{RBD}(p=0.0253)$ (Figure 1). These data indicate that $\mathrm{PD}$ patients with potential RBD have worse motor findings than those unlikely to have RBD.

Apart from motor findings, the association between depression and sleep issues in PD has been well studied and established in the literature. ${ }^{14}$ Up to $50 \%$ of $\mathrm{PD}$ patients suffer from comorbid depression; a relationship that is associated with increased disability and reduced quality of life. ${ }^{15}$ Although RBD has not been shown to be independently associated with depression in previous studies, we decided on including depression in our predictor model on the basis of its known association with sleep and PD. ${ }^{2,14}$ Our analysis found that even when age, gender, and depression were taken into account, there is a significant independent association between RBD and motor severity in PD.

There is robust evidence associating RBD with PD based on common neurobiological and neuropathological clues. A possible explanation for an association between REM sleep and PD was given by Rye and Jankovic in 2002, ${ }^{16}$ where they hypothesized that with dopamine playing a complex role in the sleep-wake cycle, some sleep disturbances in PD might be dopamine sensitive. ${ }^{16}$ Therefore a larger or earlier dopamine deficit might predict both the presence of RBD and greater motor severity. Our findings corroborate that notion.
It has also been suggested that the presence of RBD in PD assumes a more extensive neurodegenerative process, ${ }^{17}$ which includes cholinergic, serotonergic, and norepinephrine networks. This would be consistent with the fact that some of these non-dopaminergic networks are involved with REM sleep.

It has been widely accepted that RBD is a prodromal condition for PD. An article by Meissner ${ }^{18}$ on the onset of PD stated that olfactory disturbances, RBD, and autonomic dysfunction may precede the onset of motor signs by up to four decades. In a study published in April 2013, Maass and Reichmann ${ }^{19}$ also reported that RBD might predate PD. However, association of RBD with motor findings in untreated PD has not been evaluated sufficiently in the literature.

One of the strengths of our study is that none of the included study patients were on PD medications at the time their data were captured for the purposes of this cross-sectional analysis. This enables us to assess the association between RBD and motor severity in PD whereby it is not confounded by PD medications.

Disease severity could potentially confound the relationship between RBD and motor severity in PD. However, the PPMI cohort only includes patients with less than 2 years of disease duration, making this cohort relatively homogeneous from the standpoint of disease severity.

Another major advantage of our analysis is dopamine imaging confirmation of all PD cases. It is correct that some other dopaminergic neurodegenerative diseases may also produce an abnormal scan that cannot distinguish between these entities; however, this technique certainly augments the accuracy of the clinical diagnosis of PD and therefore enhances the likelihood that other movement disorders, such as essential tremor, dystonic tremor, and secondary forms of parkinsonism are excluded.

One of the limitations of the study is that the full range of motor severity cannot be represented in a newly diagnosed sample at baseline 
and the generalizability to more severe stages is questionable. Longitudinal studies may help further elucidate the association between motor PD and RBD.

The cohort used for our analysis is missing information on gender in 24 participants $(5.74 \%)$. We assume that such a small fraction of randomly missing data on gender should have no bearing on our analyses. However, we do acknowledge it to be a limitation.

Another potential limitation is that the PPMI cohort could be slightly skewed toward tremor-predominant cases, and the possible slight over-representation of tremor-predominant over non-tremorpredominant cases in this cohort relative to the general PD population will have to be taken into account. Previous studies have acknowledged the high prevalence of tremor as the initial symptom in PD patients. ${ }^{20}$ A preliminary analysis conducted by us on the baseline clinical characteristics of PPMI study patients shows similar results.

The PPMI study does not have data on the input of bed partners of the participants. Since the RBD screening questionnaire involves questions regarding hurting one's bed partner and dream enactment, this could be considered a potential limitation of the study.

Other potential limitations of this investigation include relevance and validity of the tools used for RBD and depression. Our analysis defines "likely RBD" using the screening cut-off guidelines for the REM sleep behavior disorder screening questionnaire by StiasnyKolster et. al. ${ }^{10}$ The cut-off has excellent sensitivity (0.96) but low specificity (0.56) for patients who present to the clinic. Therefore, while this gives a fairly good indication of the condition, it primarily serves as an RBD screening tool and not a diagnostic tool that calls for clinical history of dream enactment and evidence of REM sleep without atonia on a polysomnography (which involves a more complex level of financial and technical resources). However, the REM sleep behavior screening questionnaire fares better when applied to the general population, i.e., who were not seen at a sleep center, with a specificity of $92 \% .{ }^{10}$ There have also been questions regarding using the REM sleep behavior disorder screening questionnaire in PD patients, since it has been shown to give high values in healthy controls as well. However, the RBD questionnaire has also been validated in a cohort of PD patients by Nomura et al. ${ }^{21}$

Despite these potential limitations, Question 6 of the REM Sleep Behavior Questionnaire, "dream enactment" (e.g. evidence of talking/ swearing, sudden limb movements, gestures/complex movements, and things that fell around the bed) has been shown to be highly sensitive and specific to REM sleep behavior disorder. ${ }^{22}$ In their multicenter study involving 484 sleep clinic-based participants, with polysomnography as the gold standard, Postuma et al. ${ }^{22}$ found that the "dream enactment" question had a sensitivity of $93.8 \%$ and a specificity of $87.2 \%$ to detect RBD. However, in our sub-analysis, using Question 6 as a tool for classification of RBD, this correlation was not statistically significant ( $p=0.171,95 \%$ CI: $0.4884-1.135)$.

To confirm and further evaluate the relationship in future studies, we recommend employing questionnaires more specific for RBD, like the Mayo Sleep Questionnaire, ${ }^{23}$ and confirmatory polysomnography in participants screened to have RBD by the RBD questionnaire in this analysis. Additional analysis could be done to further delineate the relationship between both clinical (i.e., frequency and severity of dream enactment episodes) as well as polysomnography RBD characteristics and PD motor signs.

Age, gender, and depression have been previously associated with motor symptoms in PD. ${ }^{24-26}$ In our model, we included age, gender, GDS scores, and REM Sleep Behavior Disorder Questionnaire score as dependent variables for the outcome, MDS-UPDRS III score categories. It may be important to include more demographic and other dependent variables that could potentially affect outcome variables such as socioeconomic status, educational status, usage of antidepressants, presence of bed partners, current and past alcohol use, presence of other chronic and psychiatric disorders, etc., in future analyses.

Our analysis indicates that RBD could be a valuable target for further studies aimed at interventions to improve motor findings and functioning in PD patients. Further research aimed at understanding prodromal symptoms of PD and evaluating their relationship with motor manifestations will contribute to our understanding of prodromal pathology and will ultimately help efforts towards prevention.

\section{References}

I. Dorsey ER, Constantinescu R, Thompson JP, et al. Projected number of people with Parkinson disease in the most populous nations, 2005 through 2030. Neurology 2007;68:384-386, doi: http://dx.doi.org/10.1212/01.wnl. 0000247740.47667.03.

2. Parkinson's Outcome Project: Report to the Community. http://www. parkinson.org/NationalParkinsonFoundation/files/3f/3fea24f4-9081-4f82b96e-7a996d2133a3.pdf.

3. Postuma RB, Aarsland D, Barone $\mathrm{P}$, et al. Identifying prodromal Parkinson's disease: Pre-motor disorders in Parkinson's disease. Mov Disord 2012;27:617-626, doi: http://dx.doi.org/10.1002/mds.24996.

4. [Sleep behavior disorder (RBD) in synucleionopaties]. Glas Srp Akad Nauka Med. 2009;50:7-15.

5. Fulda S. Idiopathic REM sleep behavior disorder as a long-term predictor of neurodegenerative disorders. EPMA $\mathcal{J}$ 2011;2:451-458, doi: http://dx.doi. org/10.1007/s13167-011-0096-8.

6. Boeve BF. REM sleep behavior disorder: Updated review of the core features, the REM sleep behavior disorder-neurodegenerative disease association, evolving concepts, controversies, and future directions. Ann NY Acad Sci 2010;1184:15-54, doi: http://dx.doi.org/10.1111/j.1749-6632.2009.05115.x.

7. Poryazova R, Oberholzer M, Baumann CR, Bassetti CL. REM sleep behavior disorder in Parkinson's disease: A questionnaire-based survey. 7 Clin Sleep Med 2013;9:55-59A.

8. The Parkinson Progression Marker Initiative (PPMI). Prog Neurobiol 2011; 95:629-635, doi: http://dx.doi.org/10.1016/j.pneurobio.2011.09.005.

9. (PPMI) PsPMI. www.ppmi-info.org.

10. Stiasny-Kolster K, Mayer G, Schafer S, Moller JC, HeinzelGutenbrunner M, Oertel WH. The REM sleep behavior disorder screening questionnaire - a new diagnostic instrument. Mov Disord 2007;22:2386-2393, doi: http://dx.doi.org/10.1002/mds.21740.

I I. Almeida OP, Almeida SA. Short versions of the geriatric depression scale: A study of their validity for the diagnosis of a major depressive episode according to 
ICD-10 and DSM-IV. Int $\mathcal{F}$ Geriatr Psychiatry 1999;14:858-865, doi: http://dx.doi. org/10.1002/(SICI)1099-1166(199910)14:10<858::AID-GPS35>3.0.CO;2-8.

12. Goetz CG, Tilley BC, Shaftman SR, et al. Movement Disorder Societysponsored revision of the Unified Parkinson's Disease Rating Scale (MDSUPDRS): Scale presentation and clinimetric testing results. Mov Disord 2008;23: 2129-2170, doi: http://dx.doi.org/10.1002/mds.22340.

13. Lin X, Cook TJ, Zabetian CP, et al. DJ-1 isoforms in whole blood as potential biomarkers of Parkinson disease. Sci Rep 2012;2:954.

14. Borek LL, Kohn R, Friedman JH. Mood and sleep in Parkinson's disease. $\mathcal{F}$ Clin Psychiatry 2006;67:958-963, doi: http://dx.doi.org/10.4088/JCP.v67n0613.

15. Ravina B, Camicioli R, Como PG, et al. The impact of depressive symptoms in early Parkinson disease. Neurology 2007;69:342-347, doi: http:// dx.doi.org/10.1212/01.wnl.0000268695.63392.10.

16. Rye DB, Jankovic J. Emerging views of dopamine in modulating sleep/ wake state from an unlikely source: PD. Neurology 2002;58:341-346, doi: http:// dx.doi.org/10.1212/WNL.58.3.341.

17. Boeve BF, Silber MH, Saper CB, et al. Pathophysiology of REM sleep behaviour disorder and relevance to neurodegenerative disease. Brain 2007; 130(Pt 11):2770-2788, doi: http://dx.doi.org/10.1093/brain/awm056.

18. Meissner WG. When does Parkinson's disease begin? From prodromal disease to motor signs. Rev Neurol (Paris) 2012;168:809-814, doi: http://dx.doi. org/10.1016/j.neurol.2012.07.004.

19. Maass A, Reichmann H. Sleep and non-motor symptoms in Parkinson's disease. F Neural Transm 2013;120:565-569, doi: http://dx.doi.org/10.1007/ s00702-013-0966-4.
20. Uitti RJ, Baba Y, Wszolek ZK, Putzke DJ. Defining the Parkinson's disease phenotype: Initial symptoms and baseline characteristics in a clinical cohort. Parkinsonism Relat Disord 2005;11:139-145, doi: http://dx.doi.org/10. 1016/j.parkreldis.2004.10.007.

2I. Nomura T, Inoue Y, Kagimura T, Uemura Y, Nakashima K. Utility of the REM sleep behavior disorder screening questionnaire (RBDSQ in Parkinson's disease patients. Sleep Med 2011;12:711-713, doi: http://dx.doi. org/10.1016/j.sleep.2011.01.015.

22. Postuma RB, Arnulf I, Hogl B, et al. A single-question screen for rapid eye movement sleep behavior disorder: A multicenter validation study. Mov Disord 2012;27:913-916, doi: http://dx.doi.org/10.1002/mds.25037.

23. Boeve BF, Molano JR, Ferman TJ, et al. Validation of the Mayo Sleep Questionnaire to screen for REM sleep behavior disorder in a communitybased sample. 7 Clin Sleep Med 2013;9:475-480.

24. Dewey RB, Jr., Taneja A, McClintock SM, et al. Motor symptoms at onset of Parkinson disease and risk for cognitive impairment and depression. Cogn Behav Neurol 2012;25:115-120, doi: http://dx.doi.org/10.1097/WNN. 0b013e31826dfd62.

25. Solla P, Cannas A, Ibba FG, et al. Gender differences in motor and nonmotor symptoms among Sardinian patients with Parkinson's disease. F Neurol Sci 2012;323:33-39, doi: http://dx.doi.org/10.1016/j.jns.2012.07.026.

26. Zhou MZ, Gan J, Wei YR, Ren XY, Chen W, Liu ZG. The association between non-motor symptoms in Parkinson's disease and age at onset. Clin Neurol Neurosurg 2013;115:2103-2107, doi: http://dx.doi.org/10.1016/j. clineuro.2013.07.027.
103) Tremor and Other Hyperkinetic Movements http://www.tremorjournal.org 